\title{
Controllable Liquid Exfoliation of Fibrous Phosphorus and Its Live-cell Imaging
}

\author{
Guoqing Zhang ${ }^{1,2}$; Danmin $\operatorname{Liu}^{1,2^{*}}$; Nan Tian 3 ; Xiuhong Wang1,4; Wujuan Yan ${ }^{5}$; \\ Zikun Huang ${ }^{1,2}$; Yongzhe Zhang ${ }^{1,2}$ \\ 1. Faculty of Materials and Manufacturing, Beijing University of Technology, Beijing 100124, China \\ 2. Key Laboratory of Advanced Functional Materials, Ministry of Education, Beijing 100124, China \\ 3. Guangxi Key Laboratory of Optical and Electronic Materials and Devices, Guilin University of \\ Technology, Guilin, Guangxi Province 541004, China \\ 4. Laboratory for Biomedical Photonics, Institute of Laser Engineering, Key Laboratory of Trans- \\ scale Laser Manufacturing Technology, Ministry of Education, Beijing 100124, China. \\ 5. Department of Energy and Resources Engineering, Peking University, Beijing 100871, China \\ KEYWORDS: fibrous phosphorus, liquid exfoliation, Hansen solubility parameters, bioimaging
}




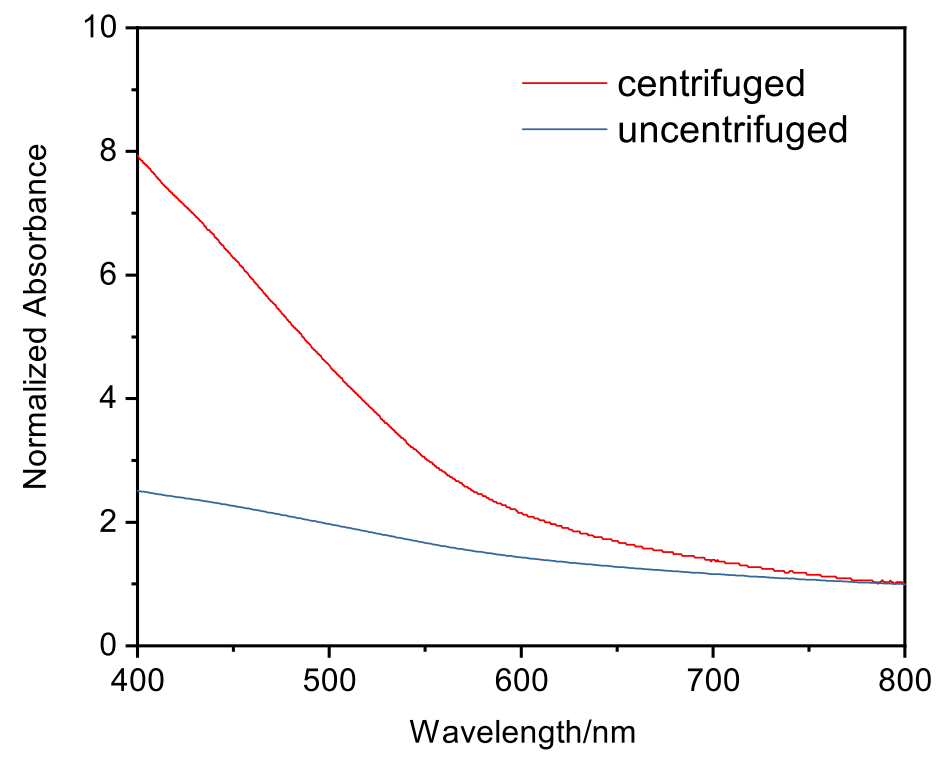

Figure S1. UV-visible spectrum of the FP dispersions with centrifuged and uncentrifuged.

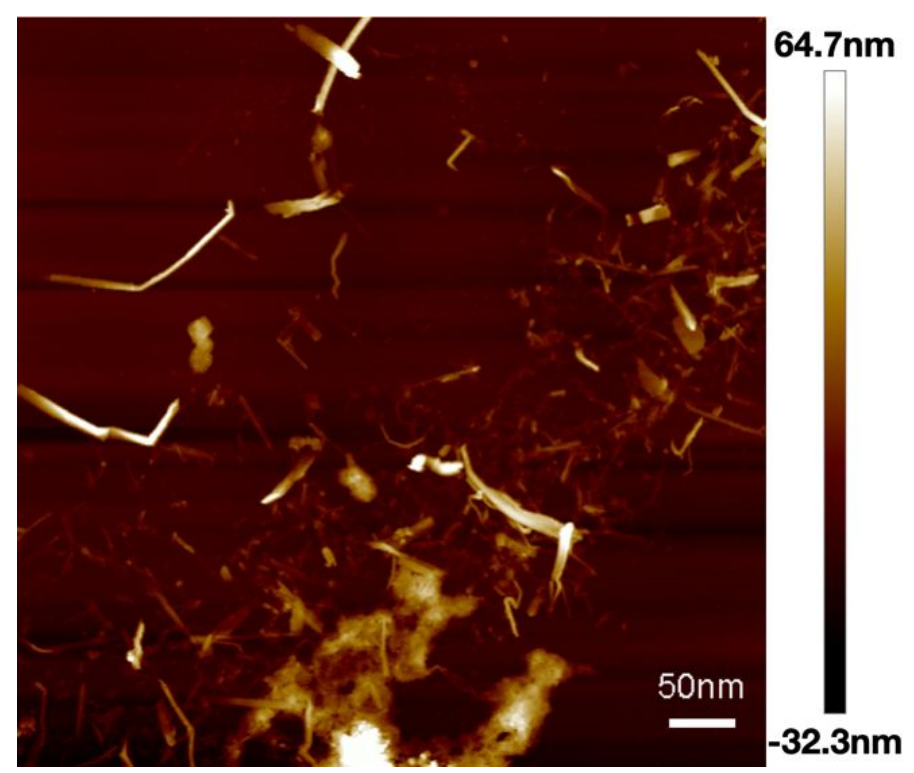

Figure S2. AFM height image of FP nanofibers. 


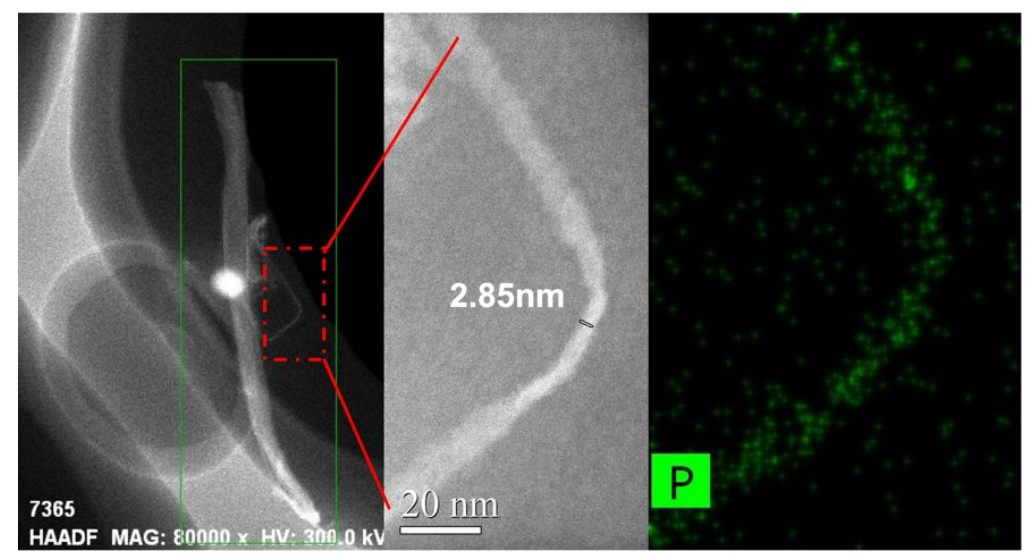

Figure S3. Bright-field transmission electron microscopy images of 2.85nm-wide FP.

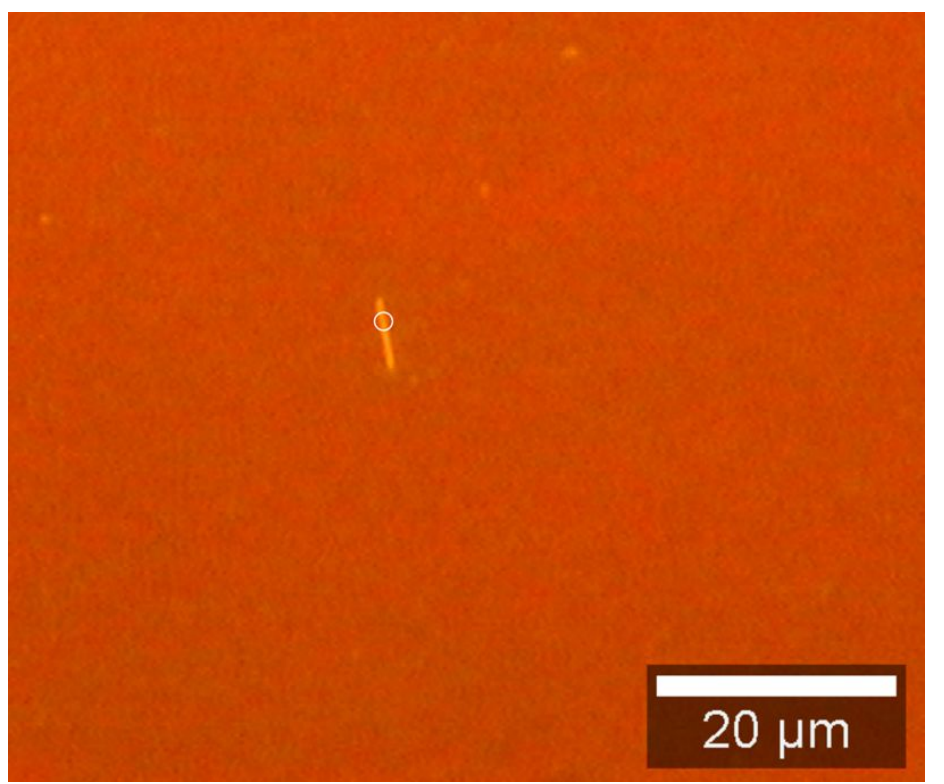

Figure S4. Optical micrograph of FP nanofiber exfoliated by acetone. The tested location is marked by a white circle.
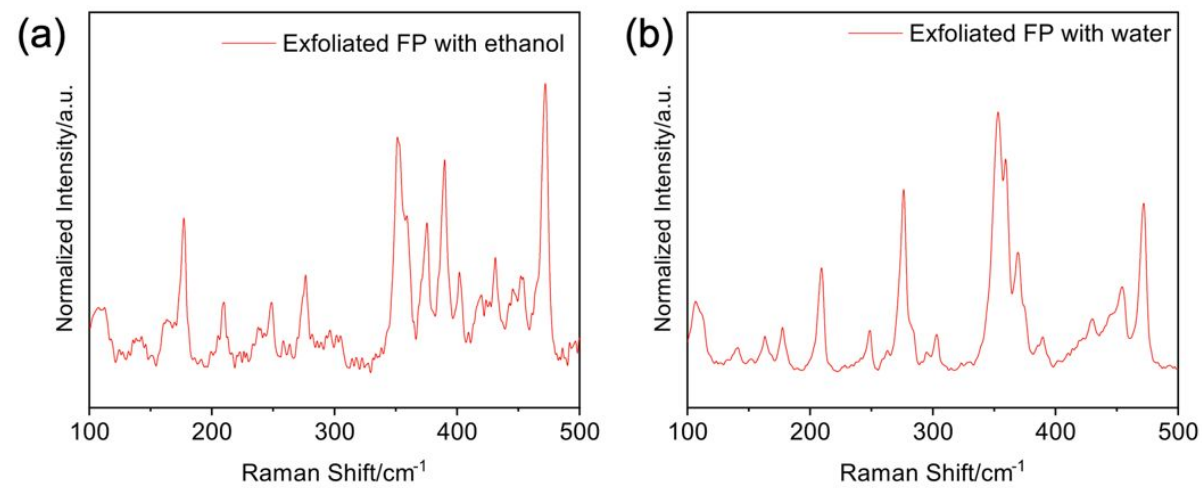

Figure S5. Raman spectrums of FP nanofiber exfoliated by (a) ethanol and (b) water. 